Man and Nature

L'homme et la nature

\title{
La vie intellectuelle et les activités culturelles à la forteresse de Louisbourg 1713-1758
}

\section{Laurent Lavoie}

Volume 4, 1985

URI : https://id.erudit.org/iderudit/1011840ar

DOI : https://doi.org/10.7202/1011840ar

Aller au sommaire du numéro

Éditeur(s)

Canadian Society for Eighteenth-Century Studies / Société canadienne d'étude du dix-huitième siècle

ISSN

0824-3298 (imprimé)

1927-8810 (numérique)

Découvrir la revue

Citer cet article

Lavoie, L. (1985). La vie intellectuelle et les activités culturelles à la forteresse de Louisbourg 1713-1758. Man and Nature / L'homme et la nature, 4, 129-138. https://doi.org/10.7202/1011840ar

Copyright (C Canadian Society for Eighteenth-Century Studies / Sociéte canadienne d'étude du dix-huitième siècle, 1985
Ce document est protégé par la loi sur le droit d'auteur. L'utilisation des services d'Érudit (y compris la reproduction) est assujettie à sa politique d'utilisation que vous pouvez consulter en ligne.

https://apropos.erudit.org/fr/usagers/politique-dutilisation/ 


\section{La vie intellectuelle et les activités culturelles à la forteresse de Louisbourg 1713-1758}

Depuis une vingtaine d'années, la reconstruction d'une partie du village de la Forteresse de Louisbourg, au Cap Breton, va bon train et de nombreuses recherches sont effectuées pour arriver à recréer le plus fidèlement possible les lieux physiques et l'ambiance qui y régnait entre 1713 et 1745 et entre 1749 et 1758 .

Grâce aux inventaires, aux lettres et aux nombreux procès, les historiens et les chercheurs parviennent à savoir qui habitait l'enceinte, comment chacun vivait, où il demeurait et même ce qu'il faisait chez lui.

Dans cette présentation, je désire souligner les divers aspects des activités culturelles à Louisbourg, suite à une lecture de plusieurs documents publiés par Parcs Canada, et essayer de voir ce que les habitants préféraient comme livres, car nous en possédons d'importantes listes.

Comme la plupart des habitants (officiers, professionnels, marchands, aubergistes, habitants-pêcheurs, artisans, pêcheurs, matelots) n'ont guère d'endroits où se rendre pour se divertir et avoir une vie intellectuelle, ils décident d'organiser à la Forteresse plusieurs activités qui leur permettent d'endurer tant soit mieux que mal les rigueurs de ce lieu stérile, loin de la mère-patrie. Pour ce faire, les villageois achètent ou apportent de nombreux livres avec eux, lorsqu'ils viennent s'installer ici; ils jouent à des jeux transportés à la Forteresse; d'autres vont tout simplement boire et 
fumer dans l'une des tavernes situées dans l'enceinte; certains préfèrent la chasse ou la promenade à cheval ou à pied; enfin, plusieurs se contentent d'assister à un encan public sur le quai pour se divertir et acheter quelques objets.

Tout d'abord parlons des livres. Vingt-cinq habitants en possèdent à la Forteresse et leur nombre s'élève à 642 , ce qui comprend également les dictionnaires et les ordonnances gouvernementales. Des vingt-cinq propriétaires, deux se situent vraiment à part, car ce sont des marchands; en effet, Jacques-François Rolland et Pierre Lambert possèdent à eux seuls quatre cent dix bouquins, surtout des abécédaires et des livres religieux.

En plus des vingt-cinq propriétaires originaux, trente-trois autres acquièrent par la suite cinq cent vingt-quatre volumes car quand quelqu'un décède, l'on vend toutes ses possessions, après en avoir fait un inventaire des plus complets. Ce qui explique le nombre restreint de livres, c'est tout d'abord les difficultés rencontrées lors des voyages maritimes, longs et parfois très accidentés, ensuite le peu de lecteurs sur place à l'arrivée. Cependant, tous s'occupent jalousement de leurs possessions et chacun peut facilement revendre sa marchandise.

Grâce aux inventaires après décès où sont notés tous les biens de la personne décédée, l'on peut constater qu'environ quatre-vingt-neuf pour cent des livres appartiennent à quarante pour cent de la population (les nobles, les officiers et les marchands). Chez les aubergistes-cabaretiers, les habitants-pêcheurs, les journaliers, les matelots et les autres, l'on ne trouve que dix-huit pour cent d'entre eux en ont.

Les livres dans les bibliothèques de la Forteresse couvrent plusieurs aspects de la pensée; d'après les inventaires, on sait pertinemment que les villageois de ce lieu aride lisent tout un éventail de livres, allant de la science jusqu'à l'art de la fortification. En effet, l'on trouve des textes dans plusieurs disciplines telles la philosophie, la médecine, la poésie, l'histoire, le droit et la magie; cependant, ceux sur la médecine sont assez rares, mais l'inventaire du chirurgien Louis Bertin et de sa femme, Henriette Lagrange, prouve bel et bien qu'il en existe.

Après les livres scientifiques, les recherches démontrent que ce sont ceux sur la religion et la philosophie qui occupent le deuxième rang. Bien sûr, la première moitié du 18e siècle est très religieuse et l'on suit assez fidèlement le calendrier de l'église catholique. Alors, on célèbre très souvent les fêtes des saints et les autres jours sacrés. Pour suppléer aux lacunes, (il n'y a point d'église et peu de prêtres pour exercer les rites religieux), la majorité des fidèles lisent chez eux plusieurs livres pieux, pour ainsi pratiquer plus ou moins leur religion. Ce n'est pas seulement l'affaire de la classe nobiliaire, mais aussi de toutes les autres.

En ce qui a trait à l'histoire, l'on trouve des oeuvres surtout sur l'épo- 
que gréco-latine et très peu sur le Moyen-Age. Par ailleurs, une quinzaine de documents juridiques font partie de la collection des imprimés de la Forteresse et une bonne partie de ces derniers meublaient la bibliothèque du parlement ou du gouvernement local.

En littérature, quelques livres seulement se trouvent sur les étagères, car les militaires ont bien d'autres chats à fouetter et ne se préoccupent guère de ce domaine. Cependant, quelques-uns en possèdent et les auteurs à la mode sont La Bruyère, Boileau, Racine et Pascal. Une bonne dizaine de titres sur les affaires militaires et marines vient s'ajouter à ce total. Mais ce qui nous surprend, c'est le peu d'importance accordée aux oeuvres de géographie, surtout dans cette région si peu connue du globe. Expliquer cette anomalie s'avère assez difficule, mais selon Gilles Groulx, ancien historien à la Forteresse, 'la géographie n'était pas très connue à cette époque-là, et en général, les oeuvres étaient mal faites'. ${ }^{1}$ Même si ce qu'il affirme est vrai, les fonctionnaires et les militaires auraient dû prendre connaissance des lieux où ils se trouvaient, en consultant des textes appropriés. Peut-être les militaires connaissent-ils les moindres détails de l'Isle Royale et qu'ils n'ont pas besoin de tels documents pour se retrouver. Peut-être aussi sont-ils plus préoccupés par la défense que par la géographie?

Selon Robert Mandrou, 'les bibliothèques de Louisbourg contiennent une immense quantité de livres bon marché' qu'il appelle' la littérature de colportage. ${ }^{2}$ Cette dernière attire évidemment les classes populaires sous l'ancien régime et les marchands Lambert et Rolland en vendent beaucoup, mais dans les inventaires on ne les mentionne point, car ils sont en général mal reliés et faits d'un mauvais papier. Dans les tavernes et auberges, on en lit un bon nombre sur tous les sujets possibles, allant de la religion à la magie, en passant par l'éducation, la pastorale et la piété.

A la Forteresse, la lecture occupe surtout les professionnels et les marchands; ces derniers, comme je l'ai mentionné ci-haut, possèdent une grande quantité de livres sur tous les sujets, car ils ont davantage le temps de lire et ils savent bien remplir leurs moments libres. Leur culture s'avère donc supérieure à celle des autres classes sociales de la population insulaire.

Peut-on identifier ces vingt-cinq propriétaires et savoir ce qu'ils ont sur leurs rayons? C'est relativement facile parce que l'on transcrivait tout lors des inventaires et des divers procès à Louisbourg.

Tout d'abord, l'ingénieur Pierre-Jérôme Boucher, qui passe près de trente-deux ans à la Forteresse entre 1717 et 1753, semble avoir des intérêts culturels très diversifiés car son choix de livres impressionne et démontre l'étendue de ses connaissances. Sur ses étagères, l'on trouve en- 
tre autres les Eléments d'Euclide par Jean-Baptiste Massillon; deux volumes de l'Art de fortifier par Vauban; deux traités du Mouvement des eaux; la Bible; le Siècle de Louis XIV par Voltaire; l'Histoire poétique et les Maximes poétiques. Non seulement s'intéresse-t-il à la littérature, mais aussi aux diverses sciences, à la religion, à la stratégie militaire et même au droit. Quel éventail.

Pour sa part, un autre ingénieur, Louis Franquet, né en Condé en 1697 et qui vient à la Forteresse entre 1750 et 1758 , lit énormément, surtout pour approfondir ses connaissances en génie. En effet, dans sa bibliothèque, on aperçoit deux tomes de l'Architecture hydraulique, six tomes des Eléments de mathématiques par Camus; l'Ingénieur pratique ou l'architecture militaire et moderne par Mr. de Lahire; les Fortifications par le Comte de Pagan; trois volumes de la Théorie et la pratique de la coupe des pierres; le Nouveau traité de toisé par Jean-Baptiste Taragon; l'Ingénieur de campagne par le chevalier Declerac; un Traité des feux artificiels et l'Esprit des Beaux-Arts. Dans sa collection inventoriée à sa mort au dix-huitième siècle, on signala également un livre sur l'art de bien gouverner, ce qui exprime sans doute son désir de briguer les suffrages pour accéder au poste de gouverneur.

Un troisième propriétaire, le dénommé Philibert Génier, né à Nantes en 1687 , habite ici dès 1717 comme habitant-pêcheur puis comme greffier du Conseil supérieur; il s'intéresse tout particulièrement à la religion et à l'histoire. D'ailleurs, à la vente de ses biens, l'on retrouve quatre tomes de la Vie des saints, l'Imitation de Jésus-Christ, le Trésor général des préservatifs, le Catéchisme, deux volumes du Journal historique et un Nouveau Testament.

Pour sa part, Blaise Lagonaire se vante d'avoir la plus belle collection dans sa bibliothèque et le plus de temps libre pour s'adonner à la lecture; c'est sa passion et son passe-temps préféré et il s'y met donc corps et âme. Son métier de marchand et commis lui permet paradoxalement de se livrer plus facilement que les autres à cette distraction enrichissante et ses intérêts s'étendent à plusieurs domaines, comme on peut le constater en vérifiant l'inventaire. En effet, il lit les classiques, la littérature satirique, les penseurs jansénistes et les traités de sciences ocultes et de magie. Ses rayons contiennent les Satires par Boileau; dix volumes de l'Histoire ancienne; deux tomes de l'Histoire de Charles XII; trois exemplaires de l'Iliade par Homère; deux volumes du Spectacle de la nature; les Pensées par Pascal; les Mémoires par le cardinal de Retz; les Oeuvres par Mme de Gomez; un dictionnaire français et la Science Curieuse ou traité de Chyromance.

Un autre commis, Jacques Delongchamps Brulay de Port Dauphin, s'adonne à la lecture pour peut-être converser avec ses compagnons. Sur 
ses étagères apparaissent certains titres déjà mentionnés comme les Caractères par Théophrastes; les Pensées par Pascal et les cinq volumes des Eléments d'Euclide, mais on observe en outre la présence des Lettres persanes par Montesquieu; l'Ordonnance de Louis XIV; la Trigonométrie; la Géométrie française, la Morale de Jésus-Christ et quatre livres sur la navigation.

Enfin, le marchand Pierre Lambert s'installe à Louisbourg en 1741 et pendant plus de quinze ans, il vend toutes sortes de livres destinés surtout à l'éducation des jeunes (les abécédaires par exemple) et à leur formation religieuse (les livres d'heures qui contenaient les prières de chaque jour). Dans son magasin, l'on retrouve les quatre tomes de la Vie des saints par le Père Jean-Baptiste Masillon, vingt-neuf alphabets pour enfants, quarante et un livres d'heures de diverses longueurs, quatre-vingts livres intitulés la Couronne de la Vierge, sept livres de piété et une bonne dizaine sur l'histoire dont l'Histoire de Charles XII par Voltaire et la Vie de Louis XIV par Nicolas Boileau et Jean Racine, en neuf tomes.

En général la majorité des propriétaires ne jouissent que d'un ou de deux livres, soit la Bible soit le catéchisme. C'est le cas de Pierre-Henry Nadau, aubergiste, de François Blondel, habitant-pêcheur, de JeanBaptiste Roussel, journalier, et de René Delaune, compagnon-pêcheur.

Selon les renseignements recueillis lors des recherches, l'on s'aperçoit que plusieurs bouquins apparaissent sur les étagères dans des langues européenes, telles l'italien et l'espagnol. Philibert Génier connaît-il le hollandais car l'un de ses volumes est écrit dans cette langue? Un cadeau peut-être ou une parure?

A l'occasion des très longues soirées d'hiver, les gens se réunissent pour discuter de ces livres lus durant la journée; ces échanges ont lieu sans aucun doute assis autour d'une bonne table où le vin coule à flots et les paroles également.

Pour bien paraître lors de ces festins, l'on s'habille luxueusement et d'une façon assez raffinée; l'on porte des chaussures à talon haut en cuir, une perruque et un costume exquis. Cependant, il ne faut pas oublier le maquillage. Pendant les allées et venues, une belle posture et une démarche gracieuse sont de rigueur. Quand on converse, il faut le faire à voix feutrée et avoir une répartie savante. La lecture aide beaucoup à étoffer le discours et voilà pourquoi plusieurs fonctionnaires et marchands s'y adonnent; ils impressionnent alors leurs voisins par leurs connaissances et leurs réparties. Cependant, comme nous l'avons mentionné plus haut, les livres ne courent pas les rues à la Forteresse et cela présente des difficultés majeures. Peut-être boit-on précisément à cause de ce phénomène?

D'autres activités culturelles existent ici, heureusement. L'une d'entre 
elles, les cartes, occupe une place primordiale surtout dans les tavernes et les auberges. En effet, l'on a découvert lors des fouilles effectuées il y a une vingtaine d'années, soixante paquets de cartes, chez l'aubergiste Grandchamps. En outre, le chevalier Johnson note, suite à une visite à Louisbourg en 1758, que 'le jeu de cartes intéresse surtout les femmes' ${ }^{3}$, ce qui l'étonne énormément.

Par contre, les hommes jouent parfois au Pharaon, une sorte de jeu de poker formellement interdit sur les lieux, mais que l'on pratique quand même dans des endroits plus ou moins secrets, malgré l'imposition de très fortes amendes à ceux qui se font prendre: entre cinq cents et mille livres.

Selon Ken Donovan, historien à la Forteresse, on joue également au solitaire et l'on en trouve une description très précise dans l'Encyclopédie de Diderot:

'nom d'un jeu qu'on a inventé depuis une cinquantaine d'années, auquel un homme peut jouer seul. C'est une tablette percée de 37 trous, disposés de manière que le premier a trois, le second cinq, les trois suivants chacun sept, le sixième cinq et le dernier trois. Tous ces trous ont chacun une cheville à la réserve d'un qui est vuide. Ce jeu consiste à prendre toutes ces chevilles les unes après les autres, en sorte qu'il n'en reste plus aucune. Elles se prennent comme on prend des dames au jeu de dames, en sautant par dessus et en se mettant à la place de celle qu'on prend et qu'on enlève. Ce jeu n'a pas grand attrait quand on en ignore la marche et n'en a point quand on la sait.'

En plus des cartes et du solitaire, certaines tables de jeux meublent les demeures des officiers ou des marchands et en lisant les inventaires on observe la présence dans ces listes de tables de 'tric-trac', de 'Picquet', de 'cadrille' et de guéridon pour jouer à la mouche.

Pour passer le temps, les soldats et les villageois peuvent se rendre à la salle publique pour jouer au billard; par contre, l'élite à la Forteresse de Louisbourg préfère s'amuser à la maison et deux habitants ont en effet chez eux une table de billard, ce qui souligne l'aisance relative de cette classe sociale dans ce coin reculé de la Nouvelle-France.

Quant aux enfants, il ne faut pas les omettre car il paraît qu'ils possèdent un vaste choix de jouets. Lors de la reconstruction, l'on découvre divers items qui tendent à le démontrer. En voici quelques exemples: un canon et un bateau miniature, des tarayettes, des boules et des sifflets de toutes sortes, des billes et des guimbardes et plusieurs autres objets.

Pour ce qui est de la musique, elle accompagne toutes les célébrations privées et publiques, les fêtes et les anniversaires. Lors de ces événements spéciaux, l'on entend surtout les instruments de musique suivants: la flûte, la guitare, la violon et la guimbarde mentionnée ci-haut. De temps 
en temps, pour souligner telle ou telle manifestation de joie, les administrateurs de Louisbourg ont recours à la musique martiale pour saluer les promeneurs dans les rues de ce village; d'habitude, l'on se rend jusqu'au quai ou jusqu'au domicile d'un officier. D'après une lettre de Robert Morgan, archiviste à l'Institut Beaton du Collège universitaire du Cap Breton ${ }^{5}$, on interprète entre autres La Diane et La Générale. Tout dernièrement, Parcs Canada vient de mettre en vente un disque de musique martiale du dix-huitième siècle.

Cependant, il faut avouer qu'une des principales occupations des habitants est la consommation d'alcool, car, entre 1713 et 1745, vingtquatre tavernes fonctionnent en moyenne. En 1734, l'on dénombre pas moins de vingt-huit propriétaires d'auberges dans le village. Bien sûr, les conséquences sont néfastes: pertes d'argent et de travail, vols, violence, maladies et malheurs.

Lors de mariages, d'anniversaires, de fêtes et du Carnaval annuel, l'on ne manque pas de profiter des provisions abondantes d'alcool; d'ailleurs, les archéologues ont découvert une énorme quantité de bouteilles de toutes formes. Pour créer de l'ambiance parmi ses officiers, le gouverneur Drucour donne presque quotidiennement des soirées, dans sa demeure, où l'alcool coule bien sûr à flots.

A l'occasion des anniversaires des administrateurs et des personnalités françaises, l'on fête également. Voici une brève liste de ces célébrations en 1752, selon l'historien Christopher Moore dans les Activités publiques à Louisbourg: ${ }^{6}$ le 17 janvier, la feste de Monseigneur Rouillé ministre de la marine'; le 13 février, c'est le tour du comte de Raymond, commandant de l'Isle Royale; le ler mai, la 'feste de M. l'Ordonnateur, Jacques Prévost'; le 28 mai, le Duc de Bourgogne lors de son ascension au trône. Toutes ces fêtes sont évidemment accompagnées de salves de canon.

Puis, le Carnaval annuel existe et c'est l'occasion pour tout le monde de s'amuser lors de ces journées interminables; les célébrations, en effet, s'éternisent parfois jusqu'aux petites heures du matin, mais aussi pendant des journées entières. Le Mardi Gras réunit le plus grand nombre de festoyeurs; cependant, certains résidents de Louisbourg reçoivent chez eux leurs invités et célèbrent le Carnaval à des dates assex incongrues.

Selon l'Ingénieur Poilly dans son Mémoire des Evénemens 7, M. Gabarus donne en 1751 un bal et un ambigu (repas froid où la viande et le dessert sont servis en même temps); le 2 février, soit cinq jours plus tard, Messieurs d'Artois font de même; puis à l'occasion du mariage de $M$. de Chauvelin, capitaine de régiment, à Mlle de Thierri, il y en a également un, le cinq février. En 1743, le riche marchand Jacques-François Rolland se plaint d'avoir dépensé quatre-vingt livres lors du Carnaval, 
car sa femme et sa belle-mère se sont livrées à des dépenses extraordinaires.

Poilly affirme qu'en 1758 le Carnaval dure du 7 janvier au mercredi des Cendres; selon lui, Mme du Bois Berthelot invite plusieurs personnes le douze janvier; cinq jours plus tard, $M$. du Souliers organise une soirée chez M. Morin; puis, c'est le tour des Las Bordes, le 23 janvier; ensuite, chez M. Gabarus, le 3 février; enfin, on festoie chez les d'Artois, le deux février et chez le gouverneur Drucour, le 31 janvier.

Dans la première moitié du dix-huitième siècle, les Français pratiquent leur foi religieuse et il en va ainsi à Louisbourg, même s'il n'existe point d'église; cependant, les Frères de la Charité de l'Ordre de St-Jean de Dieu s'occupent de l'Hôpital du Roi (où se trouvent une boulangerie, une chapelle, une cuisine, une buanderie et une morgue) et les Récollets, de religion, aidés par les Soeurs de la Congrégation de Notre-Dame (3 en 1734).

D'après John Johnston, 'A l'lle Royale et à la Forteresse, on observait les fêtes religieuses du Canada et ces jours-là était spéciaux (...). Ce sont les Récollets qui s'occupaient de la vie religieuse sous la direction bien sûr de l'évêque de Québec: Saint-Vallier (1716), Pontbriand (1745).'8

A cette occasion, l'on interdit formellement aux filles et aux garçons de danser ensemble et l'on marque ces journées-là par des processions, où la musique accompagne les marcheurs.

Lors de l'année 1744, l'on peut dénombrer plus de 26 jours sacrés célébrés par les fidèles de la Forteresse de Louisbourg; alors si on les ajoute aux autres, on arrive à un nombre considérable d'événements ou d'occasions de festoyer.

Une autre activité culturelle importante à Louisbourg est les encans qui ont lieu régulièrement dans les rues et sur le quai, à la suite de décès ou à la demande générale ou particulière.

Lors de ces encans publics, la foule en profite pour converser, jouer de la musique, s'amuser, manger et boire. Pendant ce temps-là, plusieurs vendent ou achètent des objets de tous genres; parfois, les marchandises sont légèrement endommagées ou non-désirées par les marchands euxmêmes; d'autres fois, une famille désire se séparer des biens d'un de ses membres décédé ou bien l'administration locale veut se débarrasser d'articles saisis. Pendant ces encans, certains participants arrivent à se quereller; par exemple, le 31 mai 1754, 'le huissier Michel Neel eut une dispute avec le capitaine Poullard La Bruyère de St-Malo.'9

Pour ceux qui savourent le grand air, il existe toujours les promenades le long des côtes jusqu'à Gabarus ou jusqu'à Main-à-Dieu. Sans doute, les villageois aiment-ils aussi aller sur l'eau de temps en temps pour pêcher à la ligne ou pour tout simplement se détendre. Par ailleurs, un gibier 
abondant se cache dans les bosquets tout près de la Forteresse et les chasseurs s'en donnent à coeur joie, attrapant très facilement du chevreuil, de l'orignal, de la perdrix ou du lièvre.

Dans cette enceinte perdue au bout du monde, il faut apprendre à s'occuper agréablement pour ne pas sombrer dans une angoisse profonde et l'on comprend facilement que les militaires prennent le temps de se divertir. Tous les prétextes sont bons pour le faire, même les fêtes religieuses.

Grâce aux inventaires, aux procès et aux mémoires, l'on peut en savoir beaucoup plus long sur la vie des habitants de Louisbourg. C'est à cause des inventaires, par exemple, que l'on peut se renseigner sur les bibliothèques, sur les jeux et sur l'ameublement des maisons; c'est aussi de cette façon que l'on arrive à fouiller la vie privée des propriétaires. Vivre à Louisbourg s'avérait assez difficile, mais comme la Forteresse se trouvait sur la route principale des navires marchands, ses habitants fortunés arrivaient à se procurer facilement tout ce qu'ils désiraient; en effet, la classe bourgeoise était relativement riche et grâce au commerce avec la France, les Indes, les Etats-Unis (Boston surtout) et le Canada, elle achetait des objets de luxe.

Selon l'historien Ken Donovan de Louisbourg, si l'on était éduqué, l'on avait souvent de l'argent au dix-huitième siècle; de cette façon, l'achat de livres et de cartes ne posait aucun problème et c'est pour cette raison que l'on trouve des listes impressionnantes de livres dans les inventaires. Quelques habitants bourgeois de la Forteresse en possédaient une quantité importante et cela reflète bien la puissance culturelle de la France au Siècle des Lumières, même dans cette île balayée par les vents.

\section{LAURENT LAVOIE}

Collège universitaire du Cap Breton

\section{Notes}

1 Gilles Groulx, la Bibliothèque de Louisbourg, (Parcs Canada, 1974), no. 271, p. 68.

2 Robert Mandrou, De la culture populaire aux XVII et XVIII siècles, (Paris, 1964), p. 34 .

3 Robert Morgan, Social Structure and Life in Louisbourg, Canada An Historical Magazine, vol. 1, no. 4, (Hamilton: Holt, Rinehart and Winston, 1974), p. 23. 
4 Ken Donovan, Pegboard Game Solitaire, Lettre adressée le 21 juillet 1982 à William O'Shea de la Forteresse de Louisbourg, Archives de la Forteresse de Louisbourg, 2 pages.

5 Robert Morgan, La musique martiale, Lettre adressée à M. James Boydell, Oakville, le 30 octobre 1973. Archives de la Forteresse de Louisbourg, 2 pages.

6 Christopher Moore, les Activités publiques à Louisbourg, quatre études pour les animateurs, (Parcs Canada, 1975), Archives de la Forteresse de Louisbourg, p. 58.

7 Ingénieur Poilly, Mémoire des événemens, cité par Christopher Moore in les Activités publiques à Louisbourg et par Claude Desaulniers le Carnaval de 1758 à Louisbourg, (Parcs Canada, no. 14, 1979), Archives de la Forteresse de Louisbourg.

8 A.J.B. Johnston, Religion in Life at Colonial Louisbourg 1713-1758, (Parcs Canada, 1981), Archives de la Forteresse de Louisbourg, p. 132.

9 Christopher Moore, op. cit., p. 73. 